\title{
Effect of angle of attack profiles in flapping foil propulsion
}

\author{
F.S. Hover*, Ø. Haugsdal, M.S. Triantafyllou \\ Department of Ocean Engineering, Massachusetts Institute of Technology, Building 5-228, 77Massachusetts Avenue \\ Cambridge, MA 02139-4307, USA
}

Received 30 September 2003; accepted 12 October 2003

\begin{abstract}
Propulsive performance of a harmonically heaving and pitching foil is degraded by a breakdown in the angle of attack profile, at high nondimensional frequencies. We build here upon the method employed by Read et al. (J. Fluid Structure 17 (2003) 163) to invert the key kinematic nonlinearity, and compare directly the performance obtained with four specific angle of attack profiles. These profiles are: (i) that due to simple harmonic motion in both heave and pitch motion, (ii) a square wave, (iii) a symmetric sawtooth wave, and (iv) a cosine function. The cosine angle of attack achieves a significant improvement over the other three cases, in the sense of high thrust values with reasonable efficiency. The highest thrust coefficients, however, are generally found in the sawtooth profile. Flow visualization confirms the partial or complete recovery of a reverse von Kármán wake by all three controlled profiles, concurrent with the enhanced force and efficiency at high frequencies.
\end{abstract}

(C) 2003 Elsevier Ltd. All rights reserved.

\section{Introduction}

The flow mechanism which allows propulsive forces to be created by an oscillating foil is the creation of a regular wake of vortices, which can be arranged to produce thrust or drag (Koochesfahani, 1989). The generation of leadingedge vortices (LEVs) is a mechanism that creates efficiencies which exceed predictions of the linear theory (Anderson et al., 1998), and one of the conclusions Anderson (1996) makes from DPIV experiments is that the shape of the angle of attack profile significantly influences the wake structure. Sinusoidal and square wave profiles produce the clearest wakes, with two vortices shed per cycle, whereas multiple peaks in the angle of attack profile cause additional shedding which mixes the wake, and could be expected to reduce thrust. Flapping foils as wake control devices and propulsors have been considered by many authors, including Oshima and Natsume (1980), Freymuth (1988), and Shih et al. (1992). Dynamic stall, a critical component of the foil behavior at high angles of attack, is also addressed by Ohmi et al. (1990, 1991), and reviewed by McCroskey (1982). An important and recurring observation is that vortex formation and shedding depend closely on the range and rate of angle of attack.

The use of flapping foils for biomimetic propulsion is thus complicated by the unsteady flow conditions, and we present here one of many possible steps that could be taken in the physical design and kinematic control to improve performance. We have developed a new method for controlling the angle of attack precisely under conditions where harmonic motions of the foil are inadequate. The work builds on that of Read et al. (2003), who present and demonstrate a simple method to preserve angle of attack for improved thrust performance.

The present work also follows directly the kinematic arrangements of Read et al., wherein a systematic survey of propulsive characteristics was given over the primary parameter space. This space includes the Reynolds number, the phase angle (the amount by which the pitch cycle leads the heave cycle), the ratio of heave amplitude to chord, the

\footnotetext{
*Corresponding author. Tel.: + 1-617-253-6762; fax: + 1-617-253-8125.

E-mail address: hover@mit.edu (F.S. Hover).
} 
Strouhal number or nondimensional frequency see [Triantafyllou et al. (1991)], and the maximum angle of attack taken by the foil with respect to the fluid. We consider tests with constant Reynolds number, heave to chord ratio, and phase angle; Strouhal number and maximum angle of attack are varied.

\section{Experimental setup}

\subsection{Apparatus}

All of the experiments were carried out in the MIT Department of Ocean Engineering Testing Tank. The tank itself is a $30 \mathrm{~m}$ long rectangular channel which is equipped with a two-rail carriage system. The width of the tank is $2.6 \mathrm{~m}$ and the working depth is $1.1 \mathrm{~m}$. A carriage, positioned using computer-control, carries the motion control systems for the foil as well as sensors for measuring forces and torque.

As Fig. 1 shows, the foil device consists of two streamlined aluminum struts welded to an aluminum cylinder. The foil, a NACA 0014 with constant chord of $c=10 \mathrm{~cm}$ and a span of $s=60 \mathrm{~cm}$, is constructed primarily of wood, with fiberglass cloth and resin covering. It is mounted to ball bearing assemblies between the struts; on each of the struts an acrylic circular end plate prevents flow around the ends of the foil. The gap between end plates and the foil is on the port side less than $2 \mathrm{~mm}$, and on the starboard side about $5 \mathrm{~mm}$. The mean vertical position of the foil is $0.49 \mathrm{~m}$ below the water surface.

Dynamic heaving of the entire structure shown in the figure is executed through a brushless Parker-Hanafin servomotor attached to an ALM linear table with low backlash. On the starboard strut a KISTLER 9117 three-axis piezoelectric force sensor is mounted, to measure lift and thrust forces. On the upper end of this starboard strut is mounted the pitch servo. The shaft from this pitch motor passes completely through the aluminum cylinder and is attached to a sprocket on the port side. The shaft contains two torsionally rigid flexible couplings, which allow for small misalignments between the motor and the sprocket, and a torque sensor, the KISTLER model 9065, mounted between two stainless-steel discs. The shaft is also connected to a potentiometer for angular position measurement. A chain drive connects this shaft with a lower sprocket on the port side, pivoting the foil about its $\frac{1}{3}$ chord point. The total set of signals recorded is: $X$ and $Y$ force at the starboard end of the foil, pitch torque, pitch angle (using both servo encoder and the potentiometer), and heave position (encoder).

The computer on the carriage controls the motion of the foil, through a Motion Engineering Incorporated (MEI) three-axis PID motion control card. The control code is written in $\mathrm{C}++$, and executes the heave and pitch motions as cyclic functions of the time. Data collection is accomplished using a DAS16/Jr. board and the program DASYLab. The sampling rate for the tests was $200 \mathrm{~Hz}$. All signals were digitally filtered at $30 \mathrm{~Hz}$ before any calculations and plotting. Every run was performed at least twice; all presented results show mean values.

One very important point is that we reference the thrust data by the zero created after the carriage has started its motion, before oscillation. This means that the skin friction is not included in the thrust measurement; the thrust values we report are purely the forces induced by the flapping alone.

The system was calibrated every day before testing started. Calibration was extremely consistent, with all values constant to within $1 \%$ over the days tests were made. The zero pitch position was carefully set before each set of experiments, by adjusting the pitch angle until no lift was measured.

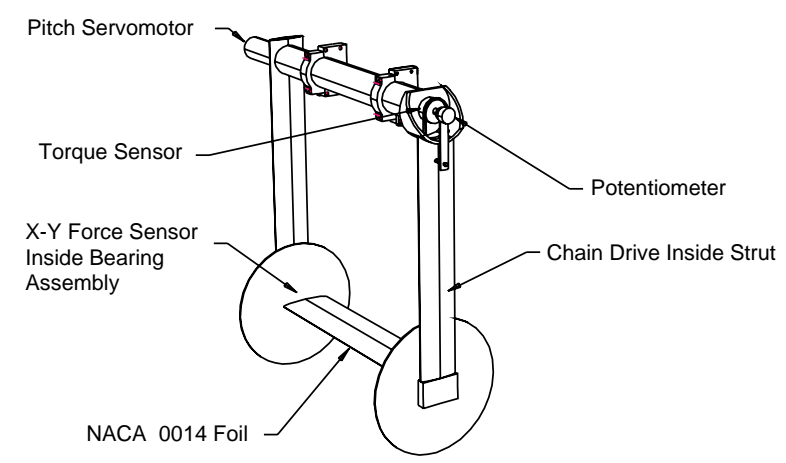

Fig. 1. The lower assembly of the flapping foil device actuates pitching of the foil, while being oscillated in the vertical direction during steady towing. 
The test matrix for each angle of attack profile was based on the following physical parameters:

(i) towing speed $U=0.3 \mathrm{~m} / \mathrm{s}$, leading to $\mathrm{Re}=U c / v \approx 30,000$;

(ii) Strouhal number $\mathrm{St}=2 h_{\mathrm{o}} f / U=[0.2-0.8]$, where $h_{\mathrm{o}}$ is peak heave amplitude and $f$ is cyclic frequency in Hertz;

(iii) heave/chord ratio $h_{\mathrm{o}} / c=1.00$;

(iv) maximum angle of attack, $\alpha_{\max }=\left[10-35^{\circ}\right]$;

(v) phase angle by which pitch leads heave, $\psi=90^{\circ}$.

\subsection{Thrust coefficient and efficiency calculations}

The average thrust force is

$$
\bar{F}_{x}=\frac{1}{T} \int_{0}^{T} X(t) \mathrm{d} t
$$

where $X(t)$ is the instantaneous force in the thrust (horizontal) direction, $T$ represents the duration of the sample window and covers an integer number of oscillations. The mean thrust coefficient is defined as follows:

$$
C_{T}=2 \bar{F}_{x} / \rho U^{2} c s
$$

where $\rho$ is the fluid density, $c$ is the foil chord, and $s$ is the span.

The efficiency, which is generally difficult to measure because of large dynamic loads in both lift and thrust directions, is defined as useful power output divided by power input:

$$
\eta=\frac{\overline{F_{x}} U}{P}
$$

where $P$ is the mean mechanical input power

$$
P=\frac{1}{T}\left(\int_{0}^{T} Y(t) \frac{\mathrm{d} h}{\mathrm{~d} t} \mathrm{~d} t+\int_{0}^{T} Q(t) \frac{d \theta}{\mathrm{d} t} \mathrm{~d} t\right)
$$

Here, $Y(t)$ is instantaneous lift (vertical direction) and $Q(t)$ is the instantaneous torque. The derivatives of heave $(\mathrm{d} h / \mathrm{d} t)$ and pitch $(\mathrm{d} \theta / \mathrm{d} t)$ positions are calculated numerically using a centered-differences scheme.

\section{Kinematics}

The main objective of this work is to control the angle of attack during flapping foil propulsion, intending to improve performance at high Strouhal numbers. We first present a brief discussion of the breakdown of angle of attack that occurs under these conditions. The net angle of attack $\alpha(t)$ seen by the foil is a combination of two angles, that induced by the physical pitch of the foil $\theta(t)$, and that due to the heave velocity; this angle is $\phi(t)=-\arctan (\dot{h}(t) / U)$, so that

$$
\alpha(t)=-\arctan (\dot{h}(t) / U)+\theta(t) .
$$

For low Strouhal numbers, harmonic $\theta(t)$ and $h(t)$ lead to nearly harmonic $\alpha(t)$, but as St increases, $\alpha(t)$ takes on additional components at a higher frequency, which degrade performance significantly in regimes of high thrust production, i.e., at high Strouhal numbers. In Read et al. (2003), we performed a power-series expansion of the tangent function to solve for higher harmonics of $h(t)$ that would flatten $\alpha(t)$. In the present work, we have extended this approach by noting that

$$
\dot{h}(t)=-U \tan (\alpha(t)-\theta(t))
$$

For a given angle of attack and pitch trajectories, the heave motion that achieves them can be obtained simply by integration of the above equation over one cycle.

In the next paragraphs four distinct angle of attack profiles are discussed in more detail: that resulting from simple harmonic heave motion (as above), a square wave angle of attack profile, which we will call the square $\alpha(t)$ profile, a symmetric sawtooth $\alpha(t)$ profile, and a cosine $\alpha(t)$ profile. In the first case, the heave motion as function of time is: $h(t)=h_{0} \sin (\omega t)$, and pitch is $\theta(t)=\theta_{0} \sin (\omega t+\psi)$. With $\psi=90^{\circ}$, the angle of attack becomes

$$
\alpha(t)=-\arctan \left(\frac{h_{0} \omega \cos (\omega t)}{U}\right)+\theta_{0} \cos (\omega t)
$$


Although variations in performance do occur with changes in $\psi$, we have generally found that $\psi=90^{\circ}$ provides a robust capability for high thrust and efficiency (Read et al., 2003). Substituting $\omega=\pi U S t / h_{0}$, we have

$$
\alpha(t)=-\arctan (\pi \mathrm{St} \cos (\omega t))+\theta_{0} \cos (\omega t) .
$$

At low Strouhal numbers, the arctangent function is inconsequential, so that $\pm \alpha_{\max }(t) \approx-\pi \mathrm{St}+\theta_{\mathrm{o}}$. Since $\alpha(t)$ in this case is comprised of two out of phase sinusoids, a given value of $\alpha_{\max }$ can be obtained with two unique values of $\theta_{0}$. One of these pertains to the thrust solution, and the other to a drag solution. At higher Strouhal numbers, the arctangent function flattens, so that the equation above represents a square wave added to an out of phase sinusoid. In this condition, small peak angles of attack simply cannot be reached because the large square wave dominates near zero crossings. Between these two extremes, the low-St solutions for $\theta_{\mathrm{o}}$ converge to a single point at a critical St, above which there is no solution. Very large values of $\alpha_{\max }$ maintain two solutions for $\theta_{\mathrm{o}}$ to large values of St, however. For example, with $h_{0} / c=1.0$, solutions for $\alpha_{\max }=10^{\circ}$ are possible for $\mathrm{St}<0.65$, whereas $\alpha_{\max }=35^{\circ}$ has solutions up to $\mathrm{St}=2.6$.

As an example case, Fig. 2 shows angle of attack profiles $\alpha(t)$ for $\alpha_{\max }=15^{\circ}$, and varying St. The profiles are very different for low and high Strouhal numbers, with the latter case inducing significant higher harmonics into the signal. The slope of $\alpha(t)$ for $\mathrm{St}=0.15$ and 0.25 changes sign twice over one period at low St, while it changes six times per cycle for higher Strouhal numbers. It is precisely these effects of the higher harmonics which are expected to degrade thrust performance, by influencing the shedding of vortices and hence the topology of the wake.

We next address finding the heave motion that, together with harmonic pitch $\theta(t)$ produces a square angle of attack profile. The desired function for the square $\alpha(t)$ profile is

$$
\alpha(t)= \begin{cases}\alpha_{\max } & \text { for } 0 \leqslant t<T / 4, \\ -\alpha_{\max } & \text { for } T / 4 \leqslant t<3 T / 4, \\ \alpha_{\max } & \text { for } 3 T / 4 \leqslant t<T .\end{cases}
$$

As shown in Fig. 3, this particular trajectory of $h(t)$ has a velocity discontinuity, and hence infinite acceleration, at the terminal points. This aspect of the prescribed heave motion physically limited the angles of attack that we could investigate accurately with our apparatus.

The function for the symmetric sawtooth $\alpha(t)$ profile is

$$
\alpha(t)= \begin{cases}\alpha_{\max }(1-4 t / T) & \text { for } 0 \leqslant t<T / 2, \\ \alpha_{\max }(4 t / T-3) & \text { for } T / 2 \leqslant t<T .\end{cases}
$$

The heave motion generating the sawtooth $\alpha(t)$ profile is much smoother than for the square angle of attack heave motion, but the peak velocities are somewhat higher.

The function for the cosine $\alpha(t)$ profile is simply:

$$
\alpha(t)=\alpha_{\max } \cos (\omega t) .
$$

The heave motion for the sawtooth and the cosine $\alpha(t)$ profiles come out to be very similar, as seen for the typical case in Fig. 3.

One important practical question that should be addressed before discussing results is why we did not try to shape the angle of attack profile by using a higher order pitch motion, instead of the heave motion. In fact, there seem to be no clear reasons not to, except that the resulting $\theta(t)$ trajectory would have to directly compensate for the effects of the arctangent, and hence could be dominated by the higher harmonics. In addition, although the torque and power consumed by the pitch degree of freedom is usually small, we believe that such pitching motions are less natural than are the heave trajectories discussed above.

\section{Results}

\subsection{Experimental conditions}

\subsubsection{Effects of towing velocity}

We first performed tests to identify a single towing speed that would maximize our ability to cover a wide range of St and $\alpha_{\max }$ with high accuracy. For this, we made an initial set of tests with $\mathrm{St}=0.45$ and $\alpha_{\max }=15^{\circ}$, and examined the thrust coefficient found at different towing speeds. With respect to the ultimate choice of $U=30 \mathrm{~cm} / \mathrm{s}$, we found the thrust coefficient was about four percent higher for $U=[35,40,50] \mathrm{cm} / \mathrm{s}$, and about 20 and six percent lower, respectively, for $U=[20,25] \mathrm{cm} / \mathrm{s}$. Thus, our tests at $\operatorname{Re} \approx 30,000$ lie at the edge separating a regime of increasing thrust 


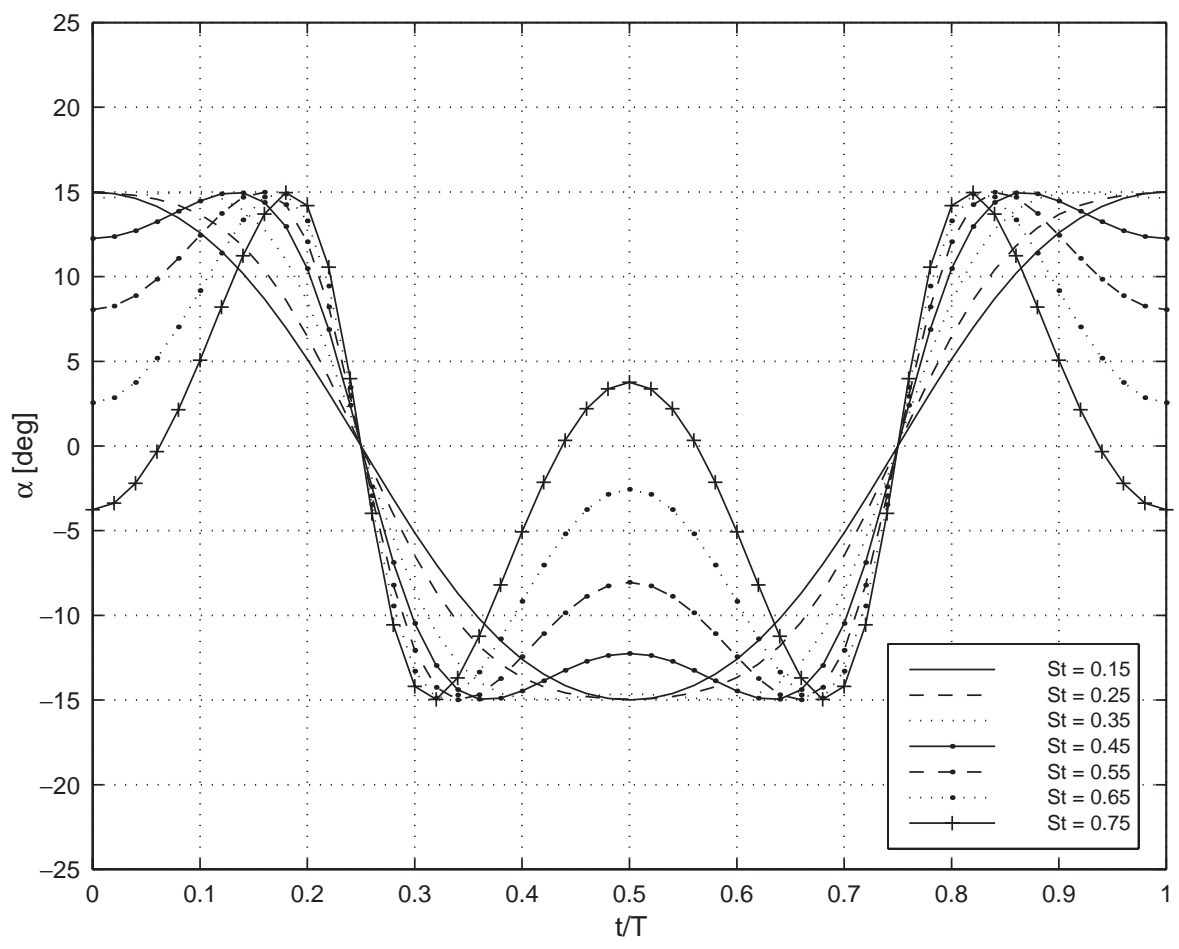

Fig. 2. Angle of attack profiles for various Strouhal numbers and $\alpha_{\max }=15^{\circ}$, showing the degradation caused by the arctangent function.
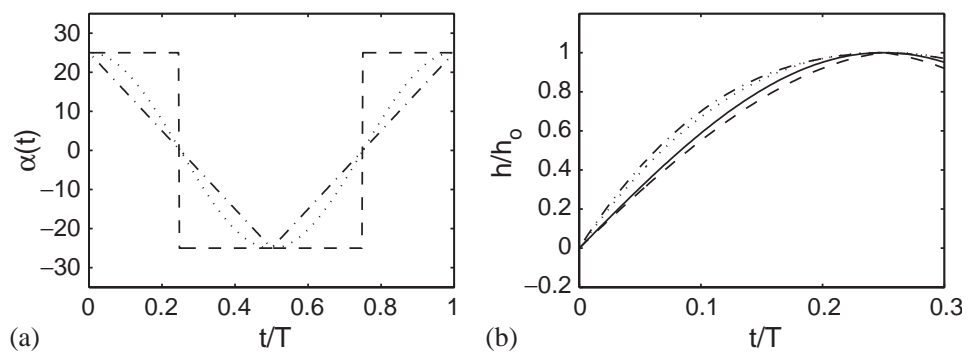

Fig. 3. (a) Angle of attack profiles under consideration: square (---), sawtooth $(\cdot-\cdot)$, and cosine $(\cdots)$. (b) Heave motions required to achieve the desired profiles, while maintaining harmonic pitch. The solid line shows harmonic heave motion.

coefficient with $U$, and a level regime. It would be an interesting extension of this short exercise to map out the explicit dependence on Reynolds number more fully.

\subsubsection{Drag properties of the stationary foil at zero angle of attack}

Because the drag of streamlined nonlifting shapes is substantially due to skin friction drag and the boundary layer is laminar for the present Reynolds number, the drag coefficient decreases with increasing Reynolds number. We performed drag tests for different Reynolds numbers in the relevant velocity region; the results are shown in Fig. 4. Compared with other drag tests of similar foils and empirical formulas, we find our drag results to be somewhat higher for the low Reynolds numbers. This we believe is due to the fact that small areas of very thin epoxy at the trailing edge were chipped off during handling. The largest imperfections were on the order of several square millimeters, viewed in planform. As the Reynolds number increases these effects are evidently reduced, and the discrepancy diminishes. For Reynolds number of $3 \times 10^{4}$, Fig. 4 shows an experimental drag coefficient of 0.0635 for our results, whereas Hoerner's (1965) empirical drag coefficient estimate is 0.04 . 


\subsubsection{Effects of finite depth}

The foil operates at a mean depth of approximately $0.5 \mathrm{~m}$, so wave-making effects must be considered, even though we did not observe substantial waves in any of our tests. Assuming that sustained wavemaking would create waves traveling at the same velocity as the carriage, we can obtain the wave number $k=g / U^{2}=109 \mathrm{rad} / \mathrm{m}$. Alternatively, supposing that the wave frequency $\omega$ matches that of our oscillations, at the lowest St we find $k \approx 6.33 \mathrm{rad} / \mathrm{m}$. The velocity and pressure fluctuations due to surface waves scale with $\mathrm{e}^{-k z}$, where $z$ is the immersion, or $0.5 \mathrm{~m}$; this factor is clearly quite small $(<0.05)$ for the present tests, so the coupling of surface effects to the foil is minor.

With regard to the interaction of shed vortices with the free surface, we found both from observation of the free surface and from dye visualization, as shown later in Fig. 9, that vortices only interact with the free surface after traveling three or more cycles downstream. Hence, the effect of this interaction on the measured forces is also expected to be minimal.

\subsection{Overall results}

Propulsion results are summarized in Figs. 5 and 6. The thrust coefficient is shown divided by $\mathrm{St}^{2}$, to facilitate graphical comparison between different cases over the full range of St. Without this normalization, the thrust curves are very steep with St.

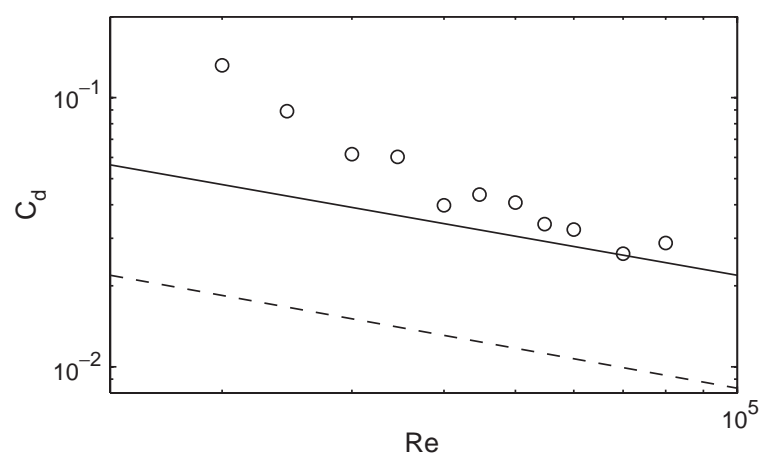

Fig. 4. Drag coefficients of the foil: ——, empirical drag coefficient (Hoerner, 1965), $C_{d}=2 C_{d f}\left(1+2 t / c+60(t / c)^{4}\right)$; - - , flat plate (Abbott and von Doenhoff, 1949), $C_{d f}=2.656 / \sqrt{\mathrm{Re}}$; $\circ$, present experiments.
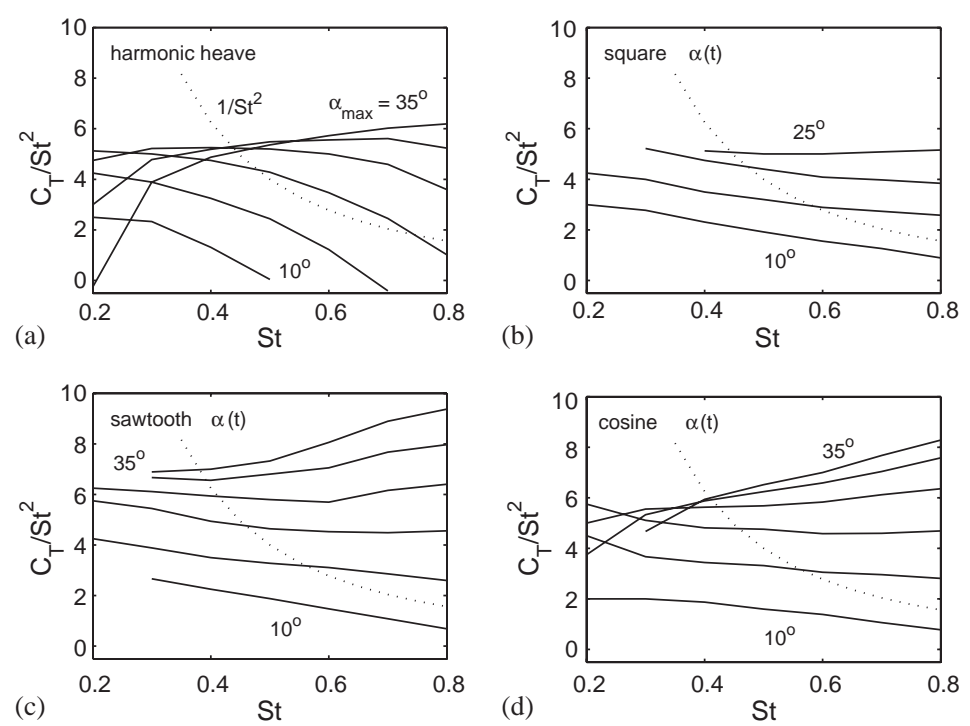

Fig. 5. Thrust coefficient $C_{T}$ (normalized with $\mathrm{St}^{2}$ ) as a function of Strouhal number St and maximum angle of attack $\alpha_{\text {max }}$. 

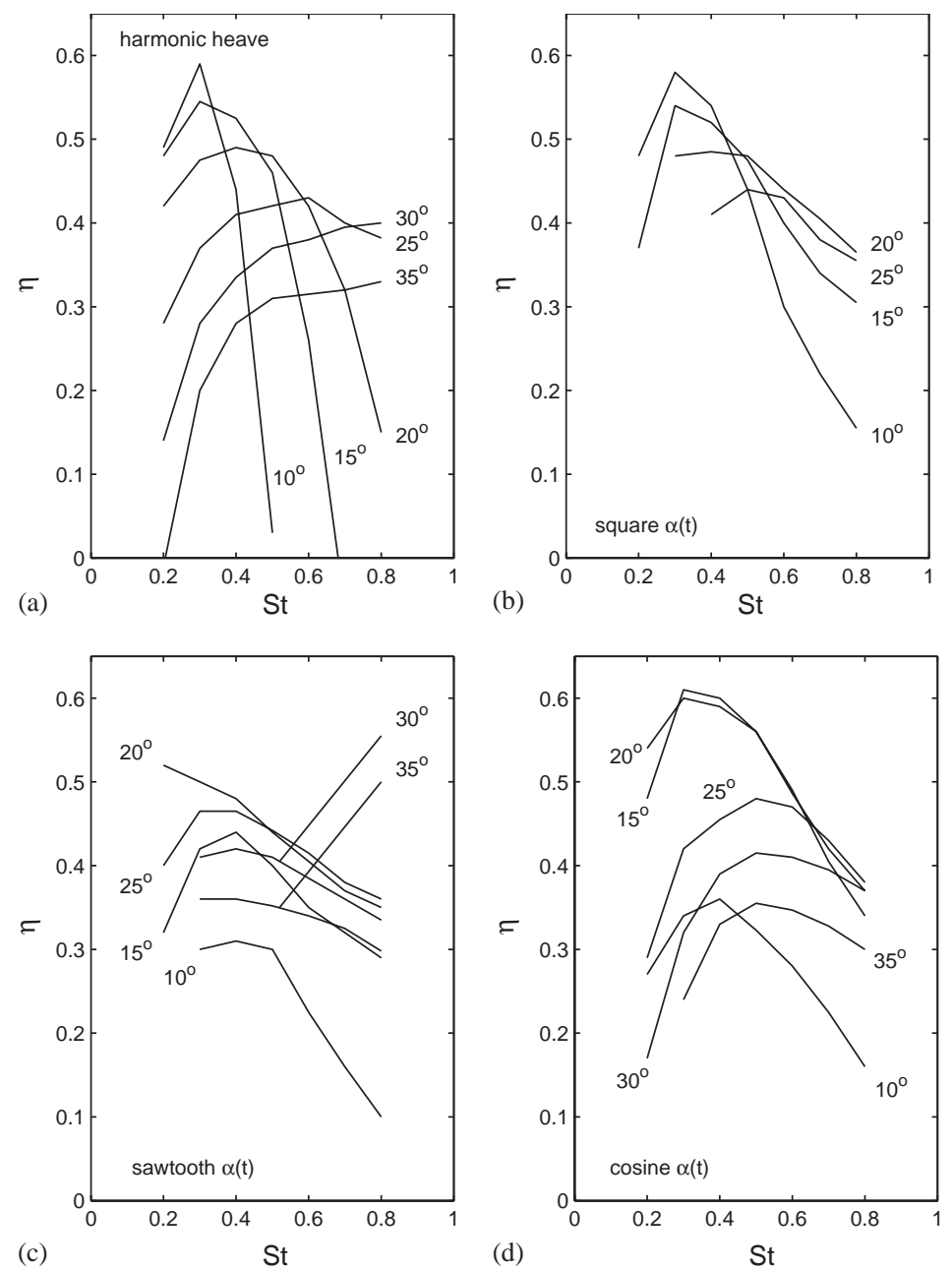

Fig. 6. Propulsive efficiency $\eta$ as a function of Strouhal number St and maximum angle of attack $\alpha_{\max }$.

\subsubsection{Harmonic heave motion}

Thrust increases from low values in the range $\mathrm{St}=0.2$ to peak values at the highest $\mathrm{St}$ tested. This thrust gradient with St is clearest at higher $\alpha_{\max }$; for lower $\alpha_{\max }$, higher harmonics degrade thrust production very substantially above $\mathrm{St}=0.5$. In contrast, efficiency $\eta$ is highest in regions of low thrust production, i.e., at lower St and low $\alpha_{\max }$. The maximum thrust coefficient is $C_{T}=3.96$, at $\alpha_{\max }=35^{\circ}$ and $\mathrm{St}=0.8$; the efficiency at this point is $\eta=0.33$. The peak efficiency found is $\eta=0.64$, for $\alpha_{\max }=10^{\circ}$ and $\mathrm{St}=0.25$. The corresponding thrust coefficient is quite low, with a value of $C_{T}=0.175$. Considering the contour of 50 percent efficiency, there exists a useful plateau of in the range $\alpha_{\max }=[10-20]^{\circ}$ and $\mathrm{St}=[0.2-0.45]$. Overall, high efficiency at low $\alpha_{\max }$ is obtained with low St, but higher efficiency at high $\alpha_{\max }$ is found at high St.

For a given $\alpha_{\max }$, the peak thrust coefficient generally occurs at the Strouhal number which creates a $30 \%$ dip in the angle of attack profile, as located in Fig. 2. With reference to the notion above that excessive changes in angle of attack slope are to be avoided, this observation is in fact a specific allowable deformation. At Strouhal numbers beyond this point, thrust rapidly decays through zero and ultimately becomes drag.

Comparing our results directly to Read et al. (2003), we see similar thrust coefficients and slightly lower efficiencies over the whole test matrix. Various experimental differences contribute to this discrepancy, including the fact that Read's foil did not have the same imperfections on the trailing edge. Furthermore, these previous tests were made at a towing velocity of $40 \mathrm{~cm} / \mathrm{s}$, and Fig. 4 shows that the experimental drag coefficient in this case is reduced from 0.0635 (the present tests) to about 0.04 . Although the first-order effects of this static drag difference are obviated by computing thrust with respect to a moving but nonoscillating foil, a speed effect may also exist during oscillating motion. In any 
event, the present set of tests employs only one foil and one towing speed, and hence direct comparisons of performance between the differing angle of attack profiles may be made.

\subsubsection{Square angle of attack profile}

The largest maximum angle of attack for this profile is $25^{\circ}$, due to limitations on the equipment. As shown in Fig. 3, the requisite heave trajectory has a discontinuity in velocity, which causes sharp transients in the physical system.

Comparing these results to the harmonic heave motion, we observe most notably that the square $\alpha(t)$ profile maintains efficiency and thrust through the full range of St, as desired. Thrust continues to climb with St along all constant $-\alpha_{\max }$ lines, and efficiency is significantly improved over the prior case. As above, low $\alpha_{\max }$ favors lower St, whereas higher $\alpha_{\max }$ favors higher St.

The maximum thrust coefficient is $C_{T}=3.16$ at $\alpha_{\max }=25^{\circ}$ and $\mathrm{St}=0.8$; the efficiency here is $\eta=0.35$. The peak efficiency is $\eta=0.60$, for $\alpha_{\max }=10^{\circ}$ and $\mathrm{St}=0.30$; the corresponding thrust coefficient is $C_{T}=0.25$.

\subsubsection{Sawtooth angle of attack profile}

The sawtooth $\alpha(t)$ profile achieves the same thrust recovery at high St as seen in the square $\alpha(t)$ profile, and achieves higher peak values, although this is at the cost of reduced efficiency overall. The maximum thrust coefficient shown is a remarkable $C_{T}=6.0$, at $\alpha_{\max }=35^{\circ}$ and $\mathrm{St}=0.8$. The corresponding efficiency here is $\eta=0.30$. This peak thrust value is significantly higher than the $C_{T}=4.0$ for the harmonic heave motion under the same condition. Similarly, at $\alpha_{\max }=25^{\circ}$, the sawtooth generates $C_{T}=4.0$ compared to $C_{T}=3.16$ for the square $\alpha(t)$ profile. Peak efficiency is $\eta_{\max }=0.53$, with a corresponding $C_{T}=0.24$, at $\alpha_{\max }=20^{\circ}$ and $\mathrm{St}=0.2$.

An interesting aspect of the efficiency curves, which is not shared in either of the two previous cases, is that peak efficiency does not occur at the lowest value of $\alpha_{\max }$ for low St. Instead, a value of $\alpha_{\max } \approx 20^{\circ}$ is favored.

\subsubsection{Cosine angle of attack profile}

As in the cases of square and sawtooth $\alpha(t)$ profiles, thrust is recovered at the highest values of St; the curves are slightly better than the square $\alpha(t)$ profile, and slightly below those of the sawtooth $\alpha(t)$ profile, especially at high St. We find a useful level of efficiency $(\eta>0.50)$ between Strouhal numbers of 0.2 and 0.6 , and $\alpha_{\max }$ in the range of $15-20^{\circ}$. The optimum efficiency point is very clear, occupying the range $\alpha_{\max }=[15,20]^{\circ}$ degrees and $\mathrm{St}=[0.3,0.4]$. As with the sawtooth $\alpha(t)$ profile, angles of attack outside this range lead to lower efficiency, with $\alpha_{\max }$ held constant.

A maximum thrust coefficient of $C_{T}=5.29$ occurs for $\alpha_{\max }=35^{\circ}$ and $\mathrm{St}=0.8$; the efficiency here is $\eta=0.31$. The peak efficiency found is $\eta=0.64$, for a $\alpha_{\max }=20^{\circ}$ and $\mathrm{St}=0.35$; the corresponding thrust coefficient is $C_{T}=0.624$.

An examination of the force traces during typical experiments at high St shows, as one might expect, that the square and sawtooth $\alpha(t)$ profiles both induce higher frequency forces correlated with rapid variations in $\alpha(t)$. These are due to a combination of small-scale vortical structures and excitation of resonant modes in our support structure. The cosine, in comparison, maintains a smoother force signal, indicating that fewer and larger vortices are being formed and shed, consistent with the model of a clean reverse von Kármán street.

\subsection{Wake structure}

The specific purpose of controlling the angle of attack is to vary the wake structure. We first consider as an example the transition of thrust to drag for $15^{\circ}$ maximum angle of attack in greater detail, with harmonic heave motion. Fig. 7 shows the angle of attack profiles for $\mathrm{St}=0.65$ and 0.70 and where we would expect to see vortices, based on a model of shedding at points of maximum $\dot{\alpha}(t)$. The simplified wake pattern that belongs to these angle of attack profiles is shown in Fig. 8. We see that vortices 1, 3, 4 and 6 are generating drag, as in the regular von Kármán vortex street, while vortices 2 and 5 are producing the main jet behind the foil. The drag vortices are increased in strength for growing St and hence also for increasing peak $\dot{\alpha}(t)$; the momentum of the vortices 2 and 5 , however, remains almost the same. Thus, it is the strengthening of drag vortices through increased fluctuations in the $\alpha(t)$ profile which leads to thrust degradation at high St.

This simple mechanism of thrust reduction is supported by two immediate observations. First, Anderson (1996) presents DPIV images in which the timing of vortex release with respect to the foil motion, and hence the angle of attack profile, can be deduced. These show clearly that vortices are shed near points of maximum rate of angle of attack, for cases of both two and four vortices being shed per cycle. The second observation pertains to a foil allowed only to pitch; in the case of harmonic pitching with no heave, vortices (which oppose the direction of foil rotation) are shed when the foil crosses through zero pitch, i.e., at its maximum rate. We confirmed this with our apparatus.

We performed dye visualization tests to further illustrate the effects of $\alpha(t)$ correction, using a fluorescent dye mixed with polyethylene oxide for thickening. Two runs are shown in Fig. 9, for $\alpha_{\max }=20^{\circ}$ at $\mathrm{St}=0.55$; this is a condition 


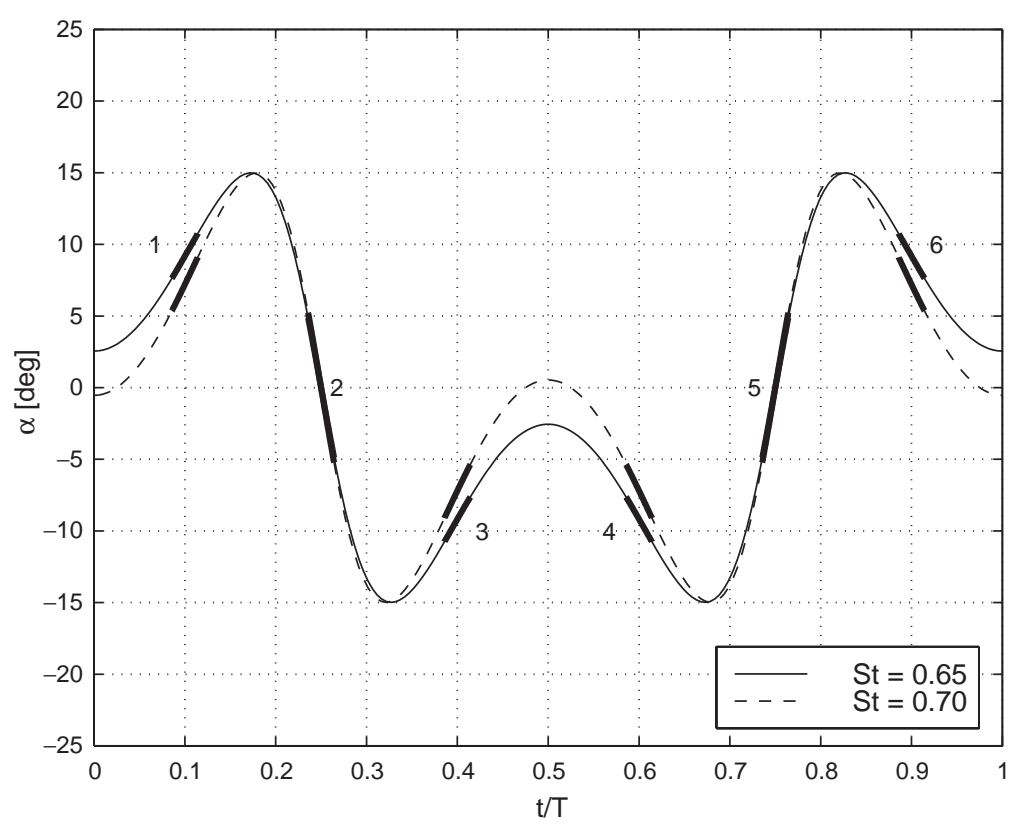

Fig. 7. Angle of attack profiles for $\mathrm{St}=0.65$ and $0.70, \alpha_{\max }=15^{\circ}$. Excess points of maximum $\mathrm{d} \alpha / \mathrm{d} t(1,3,4,6)$ correlate with additional vortices being shed into the wake.

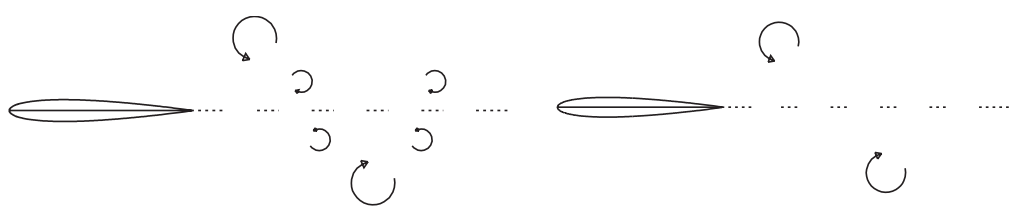

Harmonic motion

Cosine

Fig. 8. Schematized wake patterns, $\mathrm{St}=0.55$ and $\alpha_{\max }=20^{\circ}$.

where thrust with St has leveled off for the harmonic heave motion, to be followed by a rapid decrease. The measured thrust coefficient for the harmonic heave motion is $C_{T}=1.2$, and $C_{T}=1.4$ for the cosine angle of attack; efficiencies are, respectively, $\eta=0.46$ and $\eta=0.51$. The deformation of angle of attack is about $20 \%$ for the case of harmonic heave motion, and we consequently observe two dominating thrust vortices per cycle, each augmented by at least several drag vortices. The cosine $\alpha(t)$ profile, in contrast, creates a comparatively clean wake with two strong vortices per cycle.

This arrangement roughly confirms what is schematized in Fig. 8, and that fluctuations in $\alpha(t)$ indeed have a significant impact on the wake. We performed tests also at higher St and $\alpha_{\max }$, and for the other controlled profiles, which show similar ability to recover the reverse von Kármán wake.

\section{Summary and conclusions}

Force experiments show that explicit control of the angle of attack in flapping foil propulsion can broadly increase thrust and efficiency, compared to a baseline case in which the heave and pitch motions are sinusoidal. Flow visualization confirms that only two thrust vortices are produced for the cosine $\alpha(t)$ profile, even at high St. The harmonic heave motion, on the other hand, may produce six vortices for high Strouhal numbers, which corrupt the wake and ultimately cause a breakdown of the reverse von Kármán street. While the square and sawtooth $\alpha(t)$ profiles do achieve a primary goal of recovering thrust at high St, they are sharp profiles nonetheless, a trait that imposes a penalty in either or both efficiency and thrust production. 

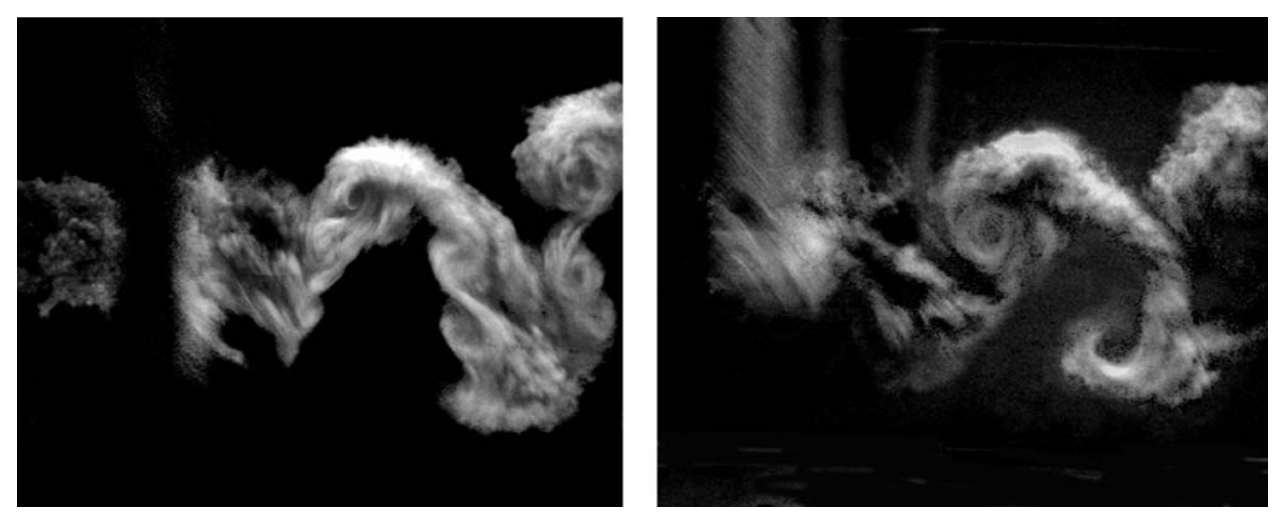

Fig. 9. Flow visualization for simple harmonic motion (left) and cosine angle of attack (right); St $=0.55, \alpha_{\max }=20^{\circ}$. The foil is at the left edge of the frame, moving toward the left.

Table 1

Maximum efficiency points $\eta_{\max }$ with corresponding thrust coefficient $C_{T}$

\begin{tabular}{lllll}
\hline Angle of attack profile & St & $\alpha_{\max }$ & $C_{T}$ & $\eta_{\max }$ \\
\hline Harmonic motion & 0.25 & 10 & 0.175 & 0.64 \\
Square $\left(\alpha_{\max } \leqslant 25^{\circ}\right)$ & 0.30 & 10 & 0.253 & 0.60 \\
Sawtooth & 0.20 & 20 & 0.243 & 0.53 \\
Cosine & 0.35 & 20 & 0.624 & 0.64 \\
\hline
\end{tabular}

Table 2

Maximum thrust coefficient points $C_{T \max }$ with corresponding efficiency $\eta$

\begin{tabular}{lllll}
\hline Angle of attack profile & St & $\alpha_{\max }$ & $C_{T \max }$ & $\eta$ \\
\hline Harmonic motion & 0.80 & 35 & 3.96 & 0.33 \\
Square $\left(\alpha_{\max } \leqslant 25^{\circ}\right)$ & 0.80 & 25 & 3.16 & 0.35 \\
Sawtooth & 0.80 & 35 & 6.02 & 0.30 \\
Cosine & 0.80 & 35 & 5.29 & 0.31 \\
\hline
\end{tabular}

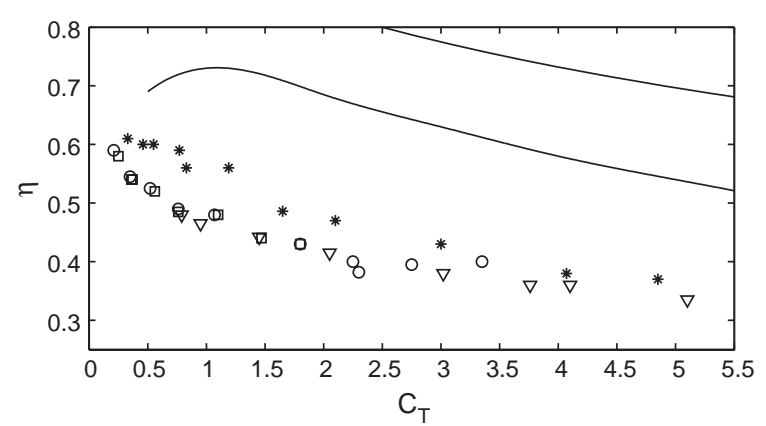

Fig. 10. Comparison of foil $C_{T}$ versus $\eta$ performance with conventional propellers. The upper solid line is the propeller ideal curve; the lower solid line is a typical open-ocean propeller curve (Lewis, 1988). The selected foil results are: $\circ$, harmonic motion; $\square$, square $\alpha(t)$; $\nabla$, sawtooth $\alpha(t) ; *$, cosine $\alpha(t)$.

In summary, Table 1 shows the maximum efficiency with corresponding thrust coefficient obtained for the different angle of attack profiles. The cosine angle of attack produces, together with the harmonic heave motion case, the highest efficiency. The cosine $\alpha(t)$ profile, however, has a much higher corresponding thrust coefficient; it is nearly four times 
larger than that of the harmonic heave motion, and more than two times larger than for the other two cases considered. In Table 2 we similarly show the maximum thrust coefficients with their corresponding efficiencies. The cosine $\alpha(t)$ profile from this point of view is slightly inferior to the sawtooth profile, which achieves higher thrust at only slightly reduced efficiency.

Considering thrust and efficiency together, as in Fig. 10, the cosine angle of attack profile achieves a very clear improvement in efficiency; the other two cases of square and sawtooth $\alpha(t)$ profiles are largely indistinguishable from the case of harmonic heave motion. The enhancement due to the cosine angle of attack is seen most dramatically in the range of $C_{T} \approx 1$, where efficiency values are increased from $\eta \approx 0.48$ to $\eta \approx 0.58$.

The best results shown here do not yet approach the thrust-efficiency capabilities of a well-designed marine propeller, also shown on this same plot (making account of the difference between our definition of $C_{T}$ using planform area, and that typically used for propellers). On the other hand, the path is open to optimization of the foil, for example through planform design to eliminate or enhance three-dimensional effects, through chordwise or spanwise flexibility, or the addition of flaps. In another paradigm entirely, we are only beginning to understand the problem of three-dimensional flapping, i.e., with a pivoted wing as found in sea turtles, birds, and insects (Dickinson, 1996).

\section{Acknowledgements}

This work was supported by the Office of Naval Research, under contract N00014-99-1-1082, monitored by Dr P Bandyopadhyay and Dr Teresa McMullen.

\section{References}

Abbott, I.H., von Doenhoff, A.E., 1949. Theory of Wing Sections. Dover, New York.

Anderson, J.M., 1996. Vorticity Control for Efficient Propulsion. Ph.D. Thesis, Massachusetts Institute of Technology and Woods Hole Oceanographic Institution.

Anderson, J.M., Streitlien, K., Barrett, D.S., Triantafyllou, M.S., 1998. Oscillating foils of high propulsive efficiency. Journal of Fluid Mechanics 360, 41-72.

Dickinson, M.H., 1996. Unsteady mechanisms of force generation in aquatic and aerial locomotion. American Zoologist 36, 537-554. Freymuth, P., 1988. Propulsive vortical signature of plunging and pitching airfoils. AIAA Journal 26, 881-883.

Hoerner, S.F., 1965. Fluid Dynamic Drag. Hoerner Fluid Dynamics, Bakersfield, CA.

Koochesfahani, M.M., 1989. Vortical patterns in the wake of an oscillating airfoil. AIAA Journal 27, 1200-1205.

Lewis, E.V. (Ed.), 1988. Principles of Naval Architecture. Society of Naval Architects and Marine Engineers, Jersey City, NJ.

McCroskey, W.J., 1982. Unsteady airfoils. Annual Review of Fluid Mechanics 14, 285-311.

Ohmi, K., Coutanceau, M., Loc, T.P., Dulieu, A., 1990. Vortex formation around an oscillating and translating airfoil at large incidences. Journal of Fluid Mechanics 211, 37-60.

Ohmi, K., Coutanceau, M., Daube, O., Loc, T.P., 1991. Further experiments on vortex formation around an oscillating and translating airfoil at large incidences. Journal of Fluid Mechanics 225, 607-630.

Oshima, Y., Natsume, A., 1980. Flow field around an oscillating airfoil. Proceedings of the Second International Symposium on Flow Visualization, Bochum, West Germany.

Read, D.A., Hover, F.S., Triantafyllou, M.S., 2003. Forces on oscillating foils for propulsion and maneuvering. Journal of Fluids and Structures 17, 163-183.

Shih, C., Lourenco, L., van Dommelen, L., Krothapalli, A., 1992. Unsteady flow past an airfoil pitching at a constant rate. AIAA Journal 30, 1153-1161.

Triantafyllou, M.S., Triantafyllou, G.S., Gopalkrishnan, R., 1991. Wake mechanics for thrust generation in oscillating foils. Physics of Fluids A 3, 2835-2837. 